\title{
GENERALIZED (U, M)-DERIVATIONS OF COMPLETELY SEMIPRIME $\Gamma$-RINGS
}

\author{
MD. MIZANOR RAHMAN ${ }^{*}$ AND AKHIL CHANDRA PAUL ${ }^{1}$ \\ Department of Mathematics, Jagannath University, Dhaka-1100, Bangladesh
}

\begin{abstract}
In this paper, the authors extend and generalize some results of previous workers to completely semiprime $\Gamma$-rings. If $U$ is an admissible Lie ideal of a completely semiprime $\Gamma$-ring $M$, $d$ is a $(U, M)$-derivation of $M$ and $\mathrm{f}$ is a generalized $(U, M)$-derivation of $M$ then under some suitable conditions, workers prove that $f(u \alpha v)=f(u) \alpha v+u \alpha d(v)$ holds for all $u, v \in U$ and $\alpha \in \Gamma$.
\end{abstract}

Key words: Admissible Lie ideal, Generalized $(U, M)$-derivation, Completely semiprime $\Gamma$-ring

\section{INTRODUCTION}

The notions of generalized derivation and Jordan generalized derivation of $\Gamma$-rings have been introduced by Ceven and Ozturk (2004). The notions of generalized derivation in rings was introduced by Hvala (1998) and Bresar (1991). Afterwards, many authors investigated comparable results on prime and semiprime rings with generalized derivtions. $(U, R)$-derivations in rings have been introduced by Faraj, Haetinger and Majeed (2010) as a generalization of Jordan derivations on a Lie ideal of a ring. Rahman and Paul (2014) introduced $(U, M)$-derivations in $\Gamma$-rings as a generalization of Jordan derivations on Lie ideals of a $\Gamma$-ring and proved that, $d(u \alpha v)=d(u) \alpha v+u \alpha d(v)$ for all $u, v \in U, \alpha \in \Gamma$ where $U$ is an admissible Lie ideal of $M$ and $d$ is a $(U, M)$ derivation of $M$. Rahman and Paul (2014) also proved that, if $u \alpha u \in U$ for all $u \in U$ and $\alpha \in \Gamma$ then $d(u \alpha m)=d(u) \alpha m+u \alpha d(m)$ for all $u \in U, m \in M$ and $\alpha \in \Gamma$. Following the notion of $(U, M)$-derivation, Rahman and Paul (2013) introduced the concept of generalized $(U, M)$-derivation and proved the analogous results considering generalized $(U, M)$-derivations of prime $\Gamma$-rings corresponding to the results of $(U, M)$-derivations. The concept of a $\Gamma$-ring was first introduced by Nobusawa (1964) and afterwards it was generalized by Barnes (1966). Many properties of $\Gamma$-rings were obtained by Barnes (1966), Kyuno (1978), Luh (1969) and others.

Let $M$ and $\Gamma$ be additive abelian groups. If there is a mapping $M \times \Gamma \times M \rightarrow M$ such that the conditions $(x+y) \alpha z=x \alpha z+y \alpha z, x(\alpha+\beta) y=x \alpha y+x \beta y, x \alpha(y+z)=x \alpha y+$ $x \alpha z$ and $(x \alpha y) \beta z=x \alpha(y \beta z)$ are satisfied for all $x, y, z \in M$ and $\alpha, \beta \in \Gamma$, then $M$ is called a $\Gamma$-ring. This concept is more general than that of a ring. A $\Gamma$-ring $M$ is

* Corresponding author : <mizanorrahman@gmail.com>.

${ }^{1}$ Department of Mathematics, University of Rajshahi, Rajshahi-6205, Bangladesh. 
semiprime if $a \Gamma M \Gamma a=0$ (with $a \in M$ ) implies $a=0$ and completely semiprime if $a \Gamma a=0$ (with $a \in M$ ) implies $a=0$. A $\Gamma$-ring $M$ is 2-torsion free if $2 a=0$ implies $a=0(a \in M)$. For any $x, y \in M$ and $\alpha \in \Gamma$, the commutator $x \alpha y-y \alpha x$ is denoted by $[x, y]_{\alpha}$. An additive subgroup $U \subseteq M$ is said to be a Lie ideal of $M$ if $u \in U, m \in M$ and $\alpha \in \Gamma$ implies $[u, m]_{\alpha} \in U$. If the Lie ideal $U$ satisfies $u \alpha u \in U$ for all $u \in U$ and $\alpha \in \Gamma$ then $\mathrm{U}$ is called square closed. $U$ is an admissible Lie ideal of $M$ if the Lie ideal $U$ is square closed and $\mathrm{U} \nsubseteq \mathrm{Z}(\mathrm{M})$, where $Z(M)$ denotes the centre of $M$. Awtar (1984) extended a well known result of Herstein (1957) to Lie ideals and proved that $d(u v)=d(u) v+u d(v)$ for all $u, v \in U$, where $\mathrm{U}(\nsubseteq \mathrm{Z})$ is a square closed Lie ideal of a 2torsion free prime ring $R$ and $d: R \rightarrow R$ is an additive mapping such that $d\left(u^{2}\right)=d(u) u+u d(u)$ holds for all $u \in U$. Ashraf and Rehman (2000) studied Lie ideals and Jordan left derivations of prime rings. Halder and Paul (2012) extended the results of Ceven (2002) to Lie ideals.

In this paper, the authors generalize some results of Rahman and Paul (2013) for admissible Lie ideal of a completely semiprime $\Gamma$-ring $M$ using the new concept of a generalized $(U, M)$-derivation of $M$. The workers require that $a \alpha b \beta c=a \beta b \alpha c$ holds for all $a, b, c \in M$ and $\alpha, \beta \in \Gamma$ (throughout the paper the authors denoted it by the symbol *) and assume that $U$ is an admissible Lie ideal of $M$.

The authors proved that, if $f$ is a generalized $(U, M)$-derivation of a completely semiprime $\Gamma$-ring $M$ with an associated $(U, M)$-derivation $d$ of $M$ and $f(a) \alpha b=f(b) \alpha a$ and $a \alpha d(b)=b \alpha d(a)$ holds for all $a, b \in U$ and $\alpha \in \Gamma$, then $f(u \alpha v)=f(u) \alpha v+u \alpha d(v)$ holds for all $u, v \in U$ and $\alpha \in \Gamma$.

Some Consequeces of Generalized (U, M)-Derivations of Completely Semiprime $\Gamma$ Rings :

Rahman and Paul (2013) introduced the concept of generalized $(U, M)$-derivation of a $\Gamma$-ring in the following way:

Definition 1. Let $U$ be a Lie ideal of a $\Gamma$-ring $M$. An additive mapping $f: M \rightarrow M$ is a generalized $(U, M)$-derivation of $M$ if there exists a $(U, M)$ derivation $d$ of $M$ such that $f(u \alpha m+s \alpha u)=f(u) \alpha m+u \alpha d(m)+f(s) \alpha u+s \alpha d(u)$ is satisfied for all $u \in U ; m, s \in M$ and $\alpha \in \Gamma$.

The following are examples of $(U, M)$-derivation and generalized $(U, M)$ derivation of a $\Gamma$-ring.

Example 1. Let $R$ be an associative ring with 1, and let $U$ be a Lie ideal of $R$. Let $M=M_{1,2}(R)$ and $\Gamma=\left\{\left(\begin{array}{c}n \cdot 1 \\ 0\end{array}\right): n \in Z\right\}$, then $M$ is a $\Gamma$-ring. 
Let $N=\{(x, x): x \in R\} \subseteq M$, then $N$ is a sub $\Gamma$-ring of $M$. Let $U_{1}=\{(u, u): u \in U\}$, then $U_{1}$ is a Lie ideal of $N$. Let $f: R \rightarrow R$ be a generalized $(U, R)$-derivation. Then there exists a $(U, R)$-derivation $d: R \rightarrow R$ such that $f(u x+s u)=f(u) x+u d(x)+f(s) u+s d(u)$ for all $u \in U, x, s \in R$. If defined a mapping $D: N \rightarrow N$ by $D((x, x))=(d(x), d(x))$, then one gets

$$
\begin{aligned}
D\left((u, u)\left(\begin{array}{l}
n \\
0
\end{array}\right)(x, x)+(y, y)\left(\begin{array}{l}
n \\
0
\end{array}\right)(u, u)\right) & =D((\text { unx }, \text { unx })+(\text { ynu, ynu })) \\
& =D((\text { unx }+y n u, u n x+y n u)) \\
& =(d(\text { unx }+y n u), d(\text { unx }+y n u)) .
\end{aligned}
$$

After some straight forward calculation, the authors get

$D\left(u_{1} \alpha x_{1}+y_{1} \alpha u_{1}\right)=D\left(u_{1}\right) \alpha x_{1}+u_{1} \alpha D\left(x_{1}\right)+D\left(y_{1}\right) \alpha u_{1}+y_{1} \alpha D\left(u_{1}\right)$,

where $u_{1}=(u, u), \quad \alpha=\left(\begin{array}{l}n \\ 0\end{array}\right), \quad x_{1}=(x, x)$ and $y_{1}=(y, y)$. Hence $D$ is a $\left(U_{1}, N\right)$ derivation on $N$. Let $F: N \rightarrow N$ be the additive mapping defined by $F((x, x))$, $=(f(x), f(x))$ then $\quad$ considering $\quad u_{1}=(u, u) \in U_{1}, \quad \alpha=\left(\begin{array}{l}n \\ 0\end{array}\right) \in \Gamma \quad$ and $\quad x_{1}=(x, x)$, $y_{1}=(y, y) \in N$, one can have

$$
\begin{aligned}
F\left(u_{1} \alpha x_{1}+y_{1} \alpha u_{1}\right)= & F((u n x+y n u, u n x+y n u)) \\
= & (f(u n x+y n u), f(\text { unx } x+y n u)) \\
= & (f(u) n x+u n d(x)+f(y) n u+y n d(u), f(u) n x+u n d(x)+f(y) n u+y n d(u)) \\
= & (f(u) n x+u n d(x), f(u) n x+u n d(x))+(f(y) n u+y n d(u), f(y) n u+y n d(u)) \\
= & (f(u) n x, f(u) n x)+(u n d(x), u n d(x))+(f(y) n u, f(y) n u)+(y n d(u), y n d(u)) \\
= & (f(u), f(u))\left(\begin{array}{l}
n \\
0
\end{array}\right)(x, x)+(u, u)\left(\begin{array}{l}
n \\
0
\end{array}\right)(d(x), d(x))+(f(y), f(y))\left(\begin{array}{l}
n \\
0
\end{array}\right)(u, u) \\
& +(y, y)\left(\begin{array}{l}
n \\
0
\end{array}\right)(d(u), d(u)) \\
= & F((u, u))\left(\begin{array}{l}
n \\
0
\end{array}\right)(x, x)+(u, u)\left(\begin{array}{l}
n \\
0
\end{array}\right)\left(D((x, x))+F((y, y))\left(\begin{array}{l}
n \\
0
\end{array}\right)(u, u)\right. \\
& +(y, y)\left(\begin{array}{l}
n \\
0
\end{array}\right) D((u, u)) . \\
\Rightarrow F\left(u_{1} \alpha x_{1}+y_{1} \alpha u_{1}\right)=F\left(u_{1}\right) \alpha x_{1}+u_{1} \alpha D\left(x_{1}\right)+F\left(y_{1}\right) \alpha u_{1}+y_{1} \alpha D\left(u_{1}\right) . &
\end{aligned}
$$

Hence $F$ is a generalized $\left(U_{1}, N\right)$-derivation on $N$.

To generalize some results of Rahman and Paul (2013) in completely semiprime $\Gamma$ rings with generalized $(U, M)$-derivations, the authors developed some important preparatory results as follows. 
Lemma 2.1 If $f$ is a generalized $(U, M)$-derivation of $M$ for which $d$ is the associated $(U, M)$-derivation of $M$. Then for all $u, v \in U ; m \in M$ and $\alpha, \beta \in \Gamma$,

$$
f(u \alpha m \beta u)=f(u) \alpha m \beta u+u \alpha d(m) \beta u+u \alpha m \beta d(u) .
$$

Proof. By the definition of generalized $(U, M)$-derivation of $M$, one obtaines $f(u \alpha m+s \alpha u)=f(u) \alpha m+u \alpha d(m)+f(s) \alpha u+s \alpha d(u) \quad$ for all $u \in U ; m, s \in M \quad$ and $\alpha \in \Gamma$.

Replacing $m$ and $s$ by $(2 u) \beta m+m \beta(2 u)$ and letting $w=u \alpha((2 u) \beta m+m \beta(2 u))+((2 u) \beta m+m \beta(2 u)) \alpha u$,

on the one hand, one gets

$$
\begin{aligned}
f(w) & =2(f(u) \alpha(u \beta m+m \beta u)+u \alpha d(u \beta m+m \beta u)+f(u \beta m+m \beta u) \alpha u+(u \beta m+m \beta u) \alpha d(u)) \\
& =2(f(u) \alpha u \beta m+f(u) \alpha m \beta u+u \alpha d(u) \beta m+u \alpha u \beta d(m)+u \alpha d(m) \beta u+u \alpha m \beta d(u) \\
& +f(u) \beta m \alpha u+u \beta d(m) \alpha u+f(m) \beta u \alpha u+m \beta d(u) \alpha u+u \beta m \alpha d(u)+m \beta u \alpha d(u)) \\
& =2(f(u) \alpha u \beta m+f(u) \alpha m \beta u+u \alpha d(u) \beta m+u \alpha u \beta d(m)+u \alpha d(m) \beta u+u \alpha m \beta d(u) \\
& +f(u) \alpha m \beta u+u \alpha d(m) \beta u+f(m) \alpha u \beta u+m \alpha d(u) \beta u+u \alpha m \beta d(u)+m \alpha u \beta d(u)) .
\end{aligned}
$$

On the other hand, one gets

$$
\begin{aligned}
f(w) & =f((2 u \alpha u) \beta m+m \beta(2 u \alpha u))+2 f(u \alpha m \beta u)+2 f(u \beta m \alpha u) \\
= & 2(f(u) \alpha u \beta m+u \alpha d(u) \beta m+u \alpha u \beta d(m)+f(m) \beta u \alpha u \\
& +m \beta d(u) \alpha u+m \beta u \alpha d(u))+4 f(u \alpha m \beta u) \\
& =2(f(u) \alpha u \beta m+u \alpha d(u) \beta m+u \alpha u \beta d(m)+f(m) \alpha u \beta u \\
& +m \alpha d(u) \beta u+m \alpha u \beta d(u))+4 f(u \alpha m \beta u)
\end{aligned}
$$

Comparing (1) and (2), and $M$ is 2-torsion free,

$f(u \alpha m \beta u)=f(u) \alpha m \beta u+u \alpha d(m) \beta u+u \alpha m \beta d(u), \forall u \in U ; m \in M ; \alpha, \beta \in \Gamma$.

Definition 2. Let $M$ be a 2-torsion free completely semiprime $\Gamma$-ring satisfying the condition (*), and $U$ be a Lie ideal of $M$. Let $f$ be a generalized $(U, M)$-derivation of $M$ with an associated $(U, M)$ - derivation $d$ of $M$. Then for all $u, v \in U$ and $\alpha \in \Gamma$, oen can define $G_{\alpha}(a, b)=f(a \alpha b)-f(a) \alpha b-a \alpha d(b)$.

Lemma 2.2 Let $M$ be 2-torsion free completely semiprime $\Gamma$-ring satisfying the condition (*), and $U$ be a Lie ideal of $M$. Let $f$ be a generalized $(U, M)$-derivation of $M$ with an associated $(U, M)$-derivation $d$ of $M$. Then for all $a, b, c \in U$ and $\alpha, \beta \in \Gamma$, the following statements hold:

(i) $G_{\alpha}(a, b)+G_{\alpha}(b, a)=0$;

(ii) $G_{\alpha}(a+b, c)=G_{\alpha}(a, c)+G_{\alpha}(b, c)$; 
(iii) $G_{\alpha}(a, b+c)=G_{\alpha}(a, b)+G_{\alpha}(a, c)$;

(iv) $G_{\alpha+\beta}(a, b)=G_{\alpha}(a, b)+G_{\beta}(a, b)$.

Lemma 2.3 Let $M$ be a 2-torsion free completely semiprime $\Gamma$-ring satisfying the condition (*), and $U$ be a Lie ideal of $M$. If $u \in U$ such that $\left[u,[u, x]_{\alpha}\right]_{\alpha}=0$ for all $x \in M$ and $\alpha \in \Gamma$, then $[u, x]_{\alpha}=0$.

Proof. The authors have $\left[u,[u, x]_{\alpha}\right]_{\alpha}=0$ for all $x \in M$ and $\alpha \in \Gamma$. For every $\beta \in \Gamma$, replacing $x$ by $x \beta x$, one obtains

$$
\begin{aligned}
0 & =\left[u,[u, x \beta x]_{\alpha}\right]_{\alpha} \\
& =\left[u, x \beta[u, x]_{\alpha}+[u, x]_{\alpha} \beta x\right]_{\alpha} \\
& =\left[u, x \beta[u, x]_{\alpha}\right]_{\alpha}+\left[u,[u, x]_{\alpha} \beta x\right]_{\alpha} \\
& =x \beta\left[u,[u, x]_{\alpha}\right]_{\alpha}+[u, x]_{\alpha} \beta[u, x]_{\alpha}+\left[u,[u, x]_{\alpha}\right]_{\alpha} \beta x+[u, x]_{\alpha} \beta[u, x]_{\alpha} \\
& =2[u, x]_{\alpha} \beta[u, x]_{\alpha} .
\end{aligned}
$$

By the 2-torsion freeness of $M$, one can obtain $[u, x]_{\alpha} \beta[u, x]_{\alpha}=0$. Since $M$ is completely semiprime, hence $[u, x]_{\alpha}=0$ for all $x \in M$ and $\alpha \in \Gamma$.

Lemma 2.4 Let $M$ be a 2-torsion free completely semiprime $\Gamma$-ring satisfying the condition (*), and $U$ be a commutative Lie ideal of $M$, then $U \subseteq Z(M)$.

Proof. Since $U$ is commutative, so one can have $\left[u,[u, x]_{\alpha}\right]_{\alpha}=0$ for all $u \in U, x \in M$ and $\alpha \in \Gamma$. Then by Lemma 2.3 , we get $[u, x]_{\alpha}=0$. This implies $U \subseteq Z(M)$.

Lemma 2.5 Let $M$ be a 2-torsion free completely semiprime $\Gamma$-ring satisfying the condition (*). If $U \neq 0$ is a sub- $\Gamma$-ring and a Lie ideal of $M$, then either $U \subseteq Z(M)$ or $U$ contains a non-zero ideal of $M$.

Proof. If $U$ is a commutative a Lie ideal of $M$, then by Lemma $2.4, U \subseteq Z(M)$. So, let $U$ be a non-commutative Lie ideal of $M$, then for some $u, v \in M$ and $\alpha \in \Gamma$, one gets $[u, v]_{\alpha} \in U$. Hence there exists an ideal I of $M$ generated by $[u, v]_{\alpha}(\neq 0)$ and $I \subseteq U$.

Lemma 2.6 Let $M$ be a 2-torsion free completely semiprime $\Gamma$-ring satisfying the condition (*). If $\mathrm{U} \nsubseteq \mathrm{Z}(\mathrm{M})$, then $Z(U)=Z(M)$.

Proof. $Z(U)$ is both a sub- $\Gamma$-ring and a Lie ideal of $M$ such that $Z(U)$ does not contain non-zero ideal of $M$. Therefore in view of Lemma 2.5, we obtain that $Z(U) \subseteq Z(M)$. Hence $Z(U)=Z(M)$.

Lemma 2.7 Let $M$ be a 2-torsion free completely semiprime $\Gamma$-ring satisfying the condition (*), and $U$ be a Lie ideal of $M$, then $Z\left([U, U]_{\Gamma}\right)=Z(U)$. 
Proof. Let $a \in M$ be any element. If $\left[a,[U, U]_{\Gamma}\right]_{\Gamma}=0$, then one can $[a, U]_{\Gamma}=0$.

Therefore, $Z\left([U, U]_{\Gamma}\right)=Z(U)$. If $[U, U]_{\Gamma} \nsubseteq \mathrm{Z}(\mathrm{M})$ then by Lemma 2.6, $a \in Z(U)$. Hence $a$ centralizes $U$. On the other hand, let $[U, U]_{\Gamma} \subseteq Z(M)$. Then one can have $\left[u,[u, a]_{\alpha}\right]_{\alpha}=0$ for all $u \in U, a \in M$ and $\alpha \in \Gamma$. Thus in view of Lemma 2.4, one obtains $[u, a]_{\alpha}=0$ for all $u \in U, a \in M$ and $\alpha \in \Gamma$. Therefore $a \in Z(U)$.

Lemma 2.8 Let $M$ be 2-torsion free completely semiprime $\Gamma$-ring satisfying the condition (*); $U$ be a Lie ideal of $M$ and $f$ be a generalized $(U, M)$-derivation of $M$ with an associated $(U, M)$-derivation $d$ of $M$. Then $G_{\alpha}(a, b) \beta[a, b]_{\alpha}+[a, b]_{\alpha} \beta G_{\alpha}(a, b)=0$ for all $a, b \in U$ and $\alpha, \beta \in \Gamma$.

Proof. For any $a, b \in U$ and $\alpha, \beta \in \Gamma$, let $w=2(a \alpha b \beta b \alpha a+b \alpha a \beta a \alpha b)$.

Using Definition1, one gets

$$
\begin{aligned}
f(w) & =f((2 a \alpha b) \beta(b \alpha a)+(b \alpha a) \beta(2 a \alpha b)) \\
& =2 f(a \alpha b) \beta(b \alpha a)+2(a \alpha b) \beta d(b \alpha a)+2 f(b \alpha a) \beta(a \alpha b)+2(b \alpha a) \beta d(a \alpha b) .
\end{aligned}
$$

On the other hand, using Lemma 2.1 one may get

$$
\begin{aligned}
f(w) & =2 f((a \alpha(b \beta b) \alpha a)+(b \alpha(a \beta a) \alpha b)) \\
& =2 f(a) \alpha(b \beta b) \alpha a+2 a \alpha d(b \beta b) \alpha a+2 a \alpha(b \beta b) \alpha d(a)+2 f(b) \alpha(a \beta a) \alpha b \\
& +2 b \alpha d(a \beta a) \alpha b+2 b \alpha(a \beta a) \alpha d(b) \\
& =2 f(a) \alpha b \beta b \alpha a+2 a \alpha d(b) \beta b \alpha a+2 a \alpha b \beta d(b) \alpha a+2 a \alpha b \beta b \alpha d(a) \\
& +2 f(b) \alpha a \beta a \alpha b+2 b \alpha d(a) \beta a \alpha b+2 b \alpha a \beta d(a) \alpha b+2 b \alpha a \beta a \alpha d(b) .
\end{aligned}
$$

Equating the two expressions for $f(w)$, one gets

$2(f(a \alpha b)-f(a) \alpha b-a \alpha d(b)) \beta b \alpha a+2(f(b \alpha a)-f(b) \alpha a-b \alpha d(a)) \beta a \alpha b+$

$2 a \alpha b \beta(d(b \alpha a)-f(b) \alpha a-b \alpha d(a))+2 b \alpha a \beta(d(a \alpha b)-f(a) \alpha b-a \alpha d(b))=0$.

Now, using Definition 2, one obtains

$2 G_{\alpha}(a, b) \beta b \alpha a+2 G_{\alpha}(b, a) \beta a \alpha b+2 a \alpha b \beta G_{\alpha}(b, a)+2 b \alpha a \beta G_{\alpha}(a, b)=0$.

Using Lemma 2.2(i), one gets

$2 G_{\alpha}(a, b) \beta b \alpha a-2 G_{\alpha}(a, b) \beta a \alpha b-2 a \alpha b \beta G_{\alpha}(a, b)+2 b \alpha a \beta G_{\alpha}(a, b)=0$.

By the 2-torsion freeness of $M$, one gets

$G_{\alpha}(a, b) \beta[a, b]_{\alpha}+[a, b]_{\alpha} \beta G_{\alpha}(a, b)=0$ for all $a, b \in U$ and $\alpha, \beta \in \Gamma$.

Lemma 2.9 Let $M$ be a 2-torsion free completely semiprime $\Gamma$-ring; $U$ be a Lie ideal of $M ; a, b \in U$ and $\alpha \in \Gamma$. If $a \alpha b+b \alpha a=0$ then $a \alpha b=b \alpha a=0$.

Proof. Suppose that $a, b \in U$ and $\alpha \in \Gamma$ such that $a \alpha b+b \alpha a=0$.

Let $\delta \in \Gamma$ be any element. Using the relation $a \alpha b=-b \alpha a$ repeatedly, one gets 


$$
\begin{aligned}
& \begin{aligned}
4(a \alpha b) \delta(a \alpha b) & =-4(b \alpha a) \delta(a \alpha b)=-4(b(\alpha a \delta) a) \alpha b \\
& =4(a(\alpha a \delta) b) \alpha b=2 a \alpha(2 a \delta b) \alpha b \\
& =-2 a \alpha(2 b \delta a) \alpha b=-4(a \alpha b) \delta(a \alpha b) .
\end{aligned} \\
& \Rightarrow 8(a \alpha b) \delta(a \alpha b)=0 .
\end{aligned}
$$

Since $M$ is 2-torsion free, so $(a \alpha b) \delta(a \alpha b)=0$. Hence, $(a \alpha b) \Gamma(a \alpha b)=0$.

By the complete semiprimeness of $M$, one gets $a \alpha b=0$. Similarly $b \alpha a=0$.

Corollary 2.1 Let $M$ be a 2-torsion free completely semiprime $\Gamma$-ring satisfying the condition (*); $U$ be a Lie ideal of $M$ and $f$ be a generalized $(U, M)$-derivation of $M$ with an associated $(U, M)$ - derivation $d$ of $M$. Then for all $a, b \in U$ and $\alpha, \beta \in \Gamma(i) G_{\alpha}(a, b) \beta[a, b]_{\alpha}=0$; (ii) $[a, b]_{\alpha} \beta G_{\alpha}(a, b)=0$.

Proof. Applying the result of Lemma 2.9 in that of Lemma 2.8, one obtains these results.

Lemma 2.10 Let $M$ be a 2-torsion free completely semiprime $\Gamma$-ring satisfying the condition (*); $U$ be a Lie ideal of $M$ and let $f$ be a generalized $(U, M)$-derivation of $M$ with an associated $(U, M)$ - derivation $d$ of $M$. Then for all $a, b, x, y \in U$ and $\alpha, \beta, \gamma \in \Gamma$ :
(i) $G_{\alpha}(a, b) \beta[x, y]_{\alpha}=0$;
(ii) $\quad[x, y]_{\alpha} \beta G_{\alpha}(a, b)=0$
(iii) $G_{\alpha}(a, b) \beta[x, y]_{\gamma}=0$;
(iv) $[x, y]_{\gamma} \beta G_{\alpha}(a, b)=0$.

Proof. (i) If one substitutes $a+x$ for $a$ in the Corollary 2.1 (i), then he gets

$$
G_{\alpha}(a+x, b) \beta[a+x, b]_{\alpha}=0 .
$$

By using Lemma 2.2 (ii),

$G_{\alpha}(a, b) \beta[a, b]_{\alpha}+G_{\alpha}(a, b) \beta[x, b]_{\alpha}+G_{\alpha}(x, b) \beta[a, b]_{\alpha}+G_{\alpha}(x, b) \beta[x, b]_{\alpha}=0$.

Now, by using Corollary 2.1(i), one obtains $G_{\alpha}(a, b) \beta[x, b]_{\alpha}+G_{\alpha}(x, b) \beta[a, b]_{\alpha}=0$.

That is, $G_{\alpha}(a, b) \beta[x, b]_{\alpha}=-G_{\alpha}(x, b) \beta[a, b]_{\alpha}$.

Now, $\left(G_{\alpha}(a, b) \beta[x, b]_{\alpha}\right) \beta\left(G_{\alpha}(a, b) \beta[x, b]_{\alpha}\right)=-G_{\alpha}(a, b) \beta[x, b]_{\alpha} \beta G_{\alpha}(x, b) \beta[a, b]_{\alpha}=0$.

Hence, by the complete semiprimeness of $M, G_{\alpha}(a, b) \beta[x, b]_{\alpha}=0$.

Similarly, by replacing $b+y$ for $b$ in this result, one gets $G_{\alpha}(a, b) \beta[x, y]_{\alpha}=0$.

(ii) Proceeding in the same way as described above by the similar replacements successively in Corollary 2.1 (ii), one obtains $[x, y]_{\alpha} \beta G_{\alpha}(a, b)=0, \forall a, b, x, y \in M$ and $\alpha, \beta \in \Gamma$.

(iii) Replacing $\alpha+\gamma$ for $\alpha$ in (i), one gets $G_{\alpha+\gamma}(a, b) \beta[x, y]_{\alpha+\gamma}=0$. 
By using Lemma 2.2(iv), $\left(G_{\alpha}(a, b)+G_{\gamma}(a, b)\right) \beta\left([x, y]_{\alpha}+[x, y]_{\gamma}\right)=0$.

This implies,

$G_{\alpha}(a, b) \beta[x, y]_{\alpha}+G_{\alpha}(a, b) \beta[x, y]_{\gamma}+G_{\gamma}(a, b) \beta[x, y]_{\alpha}+G_{\gamma}(a, b) \beta[x, y]_{\gamma}=0$.

Thus by using (i), one gets $G_{\alpha}(a, b) \beta[x, y]_{\gamma}+G_{\gamma}(a, b) \beta[x, y]_{\alpha}=0$.

That is, $G_{\alpha}(a, b) \beta[x, y]_{\gamma}=-G_{\gamma}(a, b) \beta[x, y]_{\alpha}$. Thus,

$\left(G_{\alpha}(a, b) \beta[x, y]_{\gamma}\right) \beta\left(G_{\alpha}(a, b) \beta[x, y]_{\gamma}\right)=-G_{\alpha}(a, b) \beta[x, y]_{\gamma} \beta G_{\gamma}(a, b) \beta[x, y]_{\alpha}=0$.

Hence, by the complete semiprimeness of $M$, one obtains $G_{\alpha}(a, b) \beta[x, y]_{\gamma}=0$.

(iv) By performing the similar replacement in (ii) (as in the proof of (iii)), one gets this result.

Remark 2.1 If $U$ is a commutative Lie ideal of $M$, then $U \subseteq Z(M)$. So by Definition 1 and using 2-torsion freeness of $M$, one gets $f(a \alpha b)=f(a) \alpha b+a \alpha d(b)$ for all $a, b \in U$ and $\alpha \in \Gamma$. Thus for the next results, it may be assumed that $\mathrm{U} \nsubseteq \mathrm{Z}(\mathrm{M})$.

\section{Generalized (U, M)-Derivations of Completely Semiprime $\Gamma$-Rings}

The authors proved the main results as follows:

Theorem 3.1 Let $M$ be a 2-torsion free completely semiprime $\Gamma$-ring satisfying the condition (*), $U$ be an admissible Lie ideal of $M$, and let $f$ be a generalized $(U, M)$ derivation of $\mathrm{M}$ with an associated $(U, M)$ - derivation $d$ of $M$. If $f(a) \alpha b=f(b) \alpha a$ and $a \alpha d(b)=b \alpha d(a)$ holds for all $a, b \in U$ and $\alpha \in \Gamma$, then $f(a \alpha b)=f(a) \alpha b+a \alpha d(b)$ for all $a, b \in U$ and $\alpha \in \Gamma$.

Proof. By Lemma 2.10 (iii), we have $G_{\alpha}(a, b) \beta[x, y]_{\gamma}=0$ for all $a, b, x, y \in U$ and $\alpha, \beta, \gamma \in \Gamma$. Also, by Lemma 2.10(iv), $[x, y]_{\gamma} \beta G_{\alpha}(a, b)=0$ for all $a, b, x, y \in U$ and $\alpha, \beta, \gamma \in \Gamma$.

Now $\left[G_{\alpha}(a, b),[x, y]_{\gamma}\right]_{\beta}=G_{\alpha}(a, b) \beta[x, y]_{\gamma}-[x, y]_{\gamma} \beta G_{\alpha}(a, b)=0$.

Thus, $G_{\alpha}(a, b) \subseteq Z\left([U, U]_{\Gamma}\right)=Z(U)=Z(M)$, by Lemma 2.6 and Lemma 2.7

Therefore, $G_{\alpha}(a, b) \in Z(M)$. Next, one may obtain

Therefore, one gets $2 G_{\alpha}(a, b) \beta G_{\alpha}(a, b)=G_{\alpha}(a, b) \beta f\left([a, b]_{\alpha}\right)$.

Now, by Definition 1, Lemma 2.10(iii) and 2.10(iv) with the hypothesis,

$$
\begin{aligned}
0 & =f\left(G_{\alpha}(a, b) \beta[x, y]_{\gamma}+[x, y]_{\gamma} \beta G_{\alpha}(a, b)\right) \\
& =f\left(G_{\alpha}(a, b)\right) \beta[x, y]_{\gamma}+G_{\alpha}(a, b) \beta d\left([x, y]_{\gamma}\right)+f\left([x, y]_{\gamma}\right) \beta G_{\alpha}(a, b)+[x, y]_{\gamma} \beta d\left(G_{\alpha}(a, b)\right) \\
& =2 f\left([x, y]_{\gamma}\right) \beta G_{\alpha}(a, b)+2[x, y]_{\gamma} \beta d\left(G_{\alpha}(a, b)\right) .
\end{aligned}
$$

Since $M$ is 2-torsion free, so $f\left([x, y]_{\gamma}\right) \beta G_{\alpha}(a, b)+[x, y]_{\gamma} \beta d\left(G_{\alpha}(a, b)\right)=0$. 
That is, $f\left([x, y]_{\gamma}\right) \beta G_{\alpha}(a, b)=-[x, y]_{\gamma} \beta d\left(G_{\alpha}(a, b)\right)$.

Then from (3) and (4),

$$
\begin{aligned}
2 G_{\alpha}(a, b) \beta G_{\alpha}(a, b) \beta G_{\alpha}(a, b) & =G_{\alpha}(a, b) \beta f\left([a, b]_{\alpha}\right) \beta G_{\alpha}(a, b) \\
& =-G_{\alpha}(a, b) \beta[a, b]_{\alpha} \beta d\left(G_{\alpha}(a, b)\right) \\
& =0 .
\end{aligned}
$$

That is, $2 G_{\alpha}(a, b) \beta G_{\alpha}(a, b) \beta G_{\alpha}(a, b)=0$. As $M$ is 2-torsion free, so one can have

$$
G_{\alpha}(a, b) \beta G_{\alpha}(a, b) \beta G_{\alpha}(a, b)=0 .
$$

This shows that, $G_{\alpha}(a, b)$ is a nilpotent element of the completely semiprime $\Gamma$-ring $M$, where $G_{\alpha}(a, b) \in Z(M)$. Since the centre of a completely semiprime $\Gamma$-ring does not contain any nonzero nilpotent element, so one gets $G_{\alpha}(a, b)=0$ for all $a, b \in U$ and $\alpha \in \Gamma$. Therefore, one gets $f(a \alpha b)=f(a) \alpha b+a \alpha d(b)$ for all $a, b \in U$ and $\alpha \in \Gamma$.

\section{REFERENCES}

Ashraf, M. and N. U. Rehman. 2000. On Lie ideals and Jordan left derivations of prime Rings. Arch. Math. (Brno) 36: 201-206.

Awtar, R. 1984. Lie ideals and Jordan derivations of prime rings. Amer. Math. Soc. 90(1): 9-14.

Barnes, W.E. 1966. On the $\Gamma$-rings of Nobusawa. Pacific J. Math. 18 : 411-422.

Bresar, M. 1991. On the distance of the composition of two derivations to the generalized derivations. Glasgow Math. 33: 89-93.

Ceven, Y. 2002. Jordan left derivations on completely prime gamma rings. C.U. Fen-Edebiyat Fakultesi Fen Bilimleri Dergisi. 23: 39-43.

Ceven, Y. and M. A. Ozturk. 2004. On Jordan generalized derivations in $\Gamma$-rings. Hacettepe J. Math. and Stat. 33: 11-14.

Faraj, A. K., C. Haetinger and A. H. Majeed. 2010. Generalized Higher $(U, R)$-Derivations. $J P$ Journal of Algebra 16(2): 119-142.

Halder A. K. and A. C. Paul. 2012. Jordan left derivations on Lie ideals of prime $\Gamma$-rings. Punjab University J. Math. 44: 23-29.

Hvala, B. 1998. Generalized derivations in rings. Comm. Algebra 26(4): 1147-1166.

Herstein, I. N. 1957. Jordan derivations of prime rings. Proc. Amer. Math. Soc. 8 : 1104-1110.

Kyuno, S. 1978. On prime gamma rings. Pacific J. Math. 75: 185-190.

Luh, J. 1969. On the theory of simple gamma-rings. Michigan Math. J. 16: 65-75

Nobusawa, N. 1964. On the generalizeation of the ring theory. Osaka J. Math. 1: 81-89.

Rahman, M. M. and A. C. Paul. 2013. Generalized $(U, M)(U, M)$-derivations in prime $\Gamma$-rings. Mathematical Theory and Modeling (IISTE). 3(3): 98-104.

Rahman, M. M. and A. C. Paul. 2014. ( $U$, $M$ ) derivations in prime $\Gamma$-rings. Bangladesh Journal of Scientific Research. In press. 